Artículo recibido:

29 de noviembre del 2013. Aceptado: 2 de diciembre del 2013.

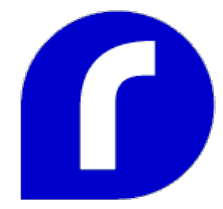

Anwar Shaikh

\section{La Primera Gran Depresión del Siglo XXI}

RESUMEN

Este artículo desarrolla la tesis según la cual la crisis financiera y la recesión internacional que se precipitaron en 2007 a partir de la crisis hipotecaria en Estados Unidos, forma parte de un movimiento de más largo plazo, el cual aquí es conceptuado como la primera gran depresión económica del siglo XXI. A fin de profundizar en esta tesis, se analiza el comportamiento de las tasas de ganancias, las tasas de interés, las remuneraciones salariales, la productividad horaria del trabajo y los procesos de endeudamiento de las familias. Se busca así caracterizar el cuadro contradictorio, inestable e insostenible que caracteriza esta situación depresiva. En el examen de las respuestas ante la crisis, se advierte respecto del sesgo dominante hacia políticas que tienden a perjudicar a las clases trabajadoras, especialmente en Europa.

Palabras Clave

\section{KEY WORDS}

Crisis capitalista, depresión económica, Capitalist crisis, economical depression, marxismo, post-keynesianismo, tasa de Marxism, post-Keynesianism, rate of ganancia, tasa de interés, deuda, profit, rate of interest, debt, recession, recesión, crisis financiera, crisis financial crisis, mortgage crisis

\section{The First Great Depression of the XXI Century}

This paper states the thesis that the first economical great depression of XXI century, was triggered by the financial crisis and global recession that began in 2007. According to its claim, this is a long-term crisis. The problem is analyzed considering the trends in the rate of profit, rate of interest, wage payments, hourly labor productivity and the process of family indebtedness. All of these aspects lead to determine the several and most outstanding traits of the depression, and its contradictory, unstable and unsustainable condition. Later on, the paper focuses its attention on the fact that the policies that are trying to solve the crisis, are at the same time, damaging life conditions of working classes, especially in Europe.
ABSTRACT hipotecaria

\begin{abstract}
Economista marxista de origen pakistaní quien ha dedicado gran parte de su obra al análisis de las teorías económicas neoclásicas y post-keynesianas. Profesor del Departamento de Economía del The New School for Social Research of New York de Estados Unidos. Sus artículos se pueden encontrar en la pagina web http://homepage.newschool.edu/ AShaikh/. Es autor, junto a E. Ahmet Tonak, de Measuring the Wealth of Nations: The Political Economy of National Accounts, publicado en 1994 por Cambridge University Press, y en castellano fue publicado por Tercer Mundo Editores con el título Valor, Acumulación y Crisis: Ensayos de Economía Política.
\end{abstract}




\section{La Primera Gran Depresión del Siglo XXI*}

La crisis económica general que se desató internacionalmente en 2008 es una Gran Depresión. Fue iniciada por una crisis financiera en EE UU, pero no fue la causa. La crisis es una fase absolutamente normal de una tendencia recurrente a largo plazo de la acumulación capitalista, en la que se suceden ondas largas expansivas y depresivas. Cuando tiene lugar la transición, la salud de la economía cambia de buena a mala. En una onda larga depresiva un choque puede desencadenar una crisis, exactamente como ocurrió con el colapso del mercado de las hipotecas subprime en 2007, y como choques anteriores desencadenaron las crisis de los 1820, 1870, 1930 y 1970 (1). En su justamente reconocida obra, La Gran Crisis de 1929, John Kenneth Galbraith señaló que si la Gran Depresión de los años 1930 fue precedida por una creciente especulación financiera, fue sin embargo el débil y frágil estado de la economía en 1929 lo que permitió que el derrumbe de los mercados bursátiles desencadenase el colapso económico (2). Lo mismo sucede hoy (3). Aquellos que prefieren considerar cada uno de estos episodios como acontecimientos excepcionales, como la casual aparición de un "cisne negro" en una bandada hasta entonces nivea (4), han olvidado la dinámica histórica que intentan explicar. $Y$ en el proceso olvidan también, convenientemente, que es la misma lógica del beneficio la que nos condena a repetir esta historia una y otra vez. reproducido con la autorización de la revista digital SinPermiso (www.sinpermiso.info). Encuentre el artículo original http://www.sinpermiso.info/ar ticulos/ficheros/XXI.pdf traducido por Gustavo Búster. 
La acumulación del capital es un proceso dinámico turbulento. Tiene poderosos ritmos endógenos que son modulados por factores cuyunturales y acontecimientos históricos concretos. El análisis de la historia real de la acumulación debe por lo tanto distinguir entre tendencias intrínsecas y su particular expresión histórica. Los ciclos económicos son el elemento más evidente de esta dinámica capitalista. Los ciclos cortos (de 3 a 5 años por los inventarios) son el resultado de la perpetua oscilación de la oferta y la demanda agregadas, y el ciclo medio (de 7 a 10 años por el capital fijo) son el producto de una fluctuación más lenta de la capacidad de producción y de la oferta agregadas (5). Pero estos ciclos económicos se apoyan en un ritmo mucho más lento por el que se suceden las ondas largas expansivas y depresivas de acumulación. Los distintos ciclos económicos se articulan en estas ondas largas de fondo (6). La historia del capitalismo siempre transcurre en un escenario en movimiento.

Tras la Gran Depresión de los años 1930 vino la Gran Stagflación de los años 1970. La crisis subyacente quedó oculta por una inflación rampante. Pero ello no impidió una perdida sustancial de puestos de trabajo, una depreciación profunda del valor real del índice de los mercados bursátiles y una ola general de quiebras bancarias e industriales. El gran temor del momento era que se desarticulasen el sistema financiero y económico (7). Baste ahora para nuestros propósitos señalar que en países como EE UU y Gran Bretaña la crisis produjo un alto nivel de paro, ataques contra el movimiento sindical, los subsidios de paro y las políticas públicas de lucha contra la pobreza, y una inflación que rápidamente erosionó tanto los salarios como el valor real de las bolsas. Otros países, como Japón, optaron por unas tasas de paro bajas y una deflación gradual de activos que prolongó la duración de la crisis, pero evitó que alcanzase la profundidad de la que sufrieron EE UU y Gran Bretaña.

Más allá de estas diferencias, en los años 1980 comenzó un nuevo boom en los principales países capitalistas, ayudado por una caída sustancial de la tasa de interés que permitió elevar considerablemente la tasa neta de ganancias, es decir, aumentó la diferencia neta entre la tasa de ganancias y la tasa de interés. La caída de la tasas de interés también facilitó la expansión del capital en todo el mundo, promovió un incremento sustancial de las deudas de consumo e infló internacionalmente las burbujas inmobiliarias y financieras. La desregulación de las actividades financieras en muchos países fue promovida por las propias entidades financieras y, con la excepción de unos pocos países como Canada, el empeño tuvo bastante éxito. Al mismo tiempo, en países como EE UU y Gran Bretaña hubo un aumento sin precedentes en la explotación de la fuerza de trabajo, a través de una reducción del crecimiento de los salarios en relación con la productividad. Como siempre, el resultado directo fue un importante aumento de la tasa de ganancias. El efecto colateral normal de una desaceleración de los salarios hubiera sido un estan- 
camiento del gasto real en consumo. Pero con unas tasas de interés decrecientes y un crédito cada vez más fácil, el gasto en consumo y en otros aspectos continuó creciendo, como una boya en un mar de deudas. Todos los límites parecían superados, todas las leyes de funcionamiento suspendidas. Y entonces se produjo la crisis y el desplome. La crisis inmobiliaria en EE UU fue solo el detonante inmediato. El problema real era que la reducción de la tasa de interés y el crecimiento de la deuda que había alimentado el boom habían alcanzado sus límites.

La actual crisis esta aun en pleno desarrollo. Se han creado enormes cantidades de dinero en todos los principales países avanzados, que se han canalizado hacia el sector privado empresarial para reactivarlo. Pero la mayor parte de este dinero se ha quedado allí. Los bancos no quieren aumentar el crédito en un clima de incertidumbre y riesgo en el que no están seguros de recuperar su dinero con una tasa de interés rentable. Otros sectores, como la industria automovilística tienen problemas similares debido al peso de los grandes inventarios de mercancías invencidas a las que necesitan dar salida antes de pensar en nuevas inversiones. Por todo ello, la mayor parte de la ciudadanía no ha visto el menor beneficio de las grandes cantidades de dinero que se han arrojado al mercado y las tasas de paro siguen altas. A este respecto, resulta cuanto menos chocante lo poco que se ha hecho para crear empleo a través de programas de inversión pública, como hizo la Administración Roosevelt en los años 1930.

Lo que plantea una pregunta fundamental: ¿cómo es posible que el sistema capitalista, cuyas instituciones, regulaciones y estructuras políticas han cambiado tanto a través de su evolución, sea capaz de reproducir ciertas tendencias económicas recurrentes? La respuesta reside en el hecho de que estas tendencias particulares nacen de la búsqueda de beneficios, que sigue siendo el regulador central de la actitud empresarial a través de la historia. La apariencia del capitalismo muta constantemente para que su naturaleza siga siendo la misma. Una explicación teórica completa de esta dinámica escapa al objetivo y las posibilidades de este articulo, pero podemos tener una buena apreciación de su lógica examinando la relación entre acumulación y beneficios. Me concentraré a continuación en EE UU porque sigue siendo el centro del mundo capitalista avanzado y donde se originó la crisis. Pero hay que advertir que las victimas son globales y sobre todo están constituidas por las mujeres, los niños y los parados que sufren en este mundo. 


\section{Acumulación y beneficios}

"El motor de toda empresa es....el beneficio" (8) J.M. Keynes

"Vender sin beneficios es absurdo" (19) Business Week

Toda empresa sabe, a riesgo de perecer, que el beneficio es su razón de existir. Los economistas clásicos defendieron que el motor de la acumulación es la diferencia entre la tasa de ganancias ( $\mathbf{r}$ ) y la tasa de interés (i). La razón es que la tasa de ganancias es el retorno de una inversión activa, mientras que la tasa de interés es el retorno de una inversión pasiva. Se puede invertir una cantidad dada de capital en la producción o venta de mercancías, en el préstamo de dinero o en la especulación. En cada uno de estos casos, el retorno es la tasa de ganancias, con todos los riesgos, incertidumbres y errores que implican estas prácticas. Como han aprendido las gentes de negocios, "hay certezas certeras, certezas desconocidas e ignorancias desconocidas" (10). Por otra parte, la misma cantidad de capital puede ser invertido sin más en una cuenta de ahorros o en bonos del tesoro, ganando un interés tranquilo y relativamente seguro. La tasa de interés es la retaguardia, la alternativa segura a la tasa de ganancias de una inversión activa. Marx defendió que es la diferencia entre las dos tasas, que llamó la tasa de beneficio empresarial $(\mathbf{r}-\mathbf{i})$, la que moviliza la inversión activa. Keynes dijo más o menos lo mismo: llamó a la tasa de ganancias la "eficiencia marginal del capital" (MEC), y se fijo en la diferencia entre el MEC y la tasa de interés como el fundamento de la viabilidad de la inversión. Tanto la economía neo-clásica como la post-keynesiana subrayan esta misma diferencia, aunque a través de un rodeo: los precios de producción son definidos de manera que incluyan un "coste de oportunidad" que comprende el interés equivalente en el capital social, de manera que el "beneficio económico" es la suma del beneficio de empresa y la correspondiente tasa de ganancias es simplemente la tasa de beneficio de la empresa (r-i).

Pongamos un ejemplo. Supongamos que el beneficio anual de la compañía es de 100.000 dólares. Supongamos que la tasa de interés en vigor es el 4\% y que el capital social de la empresa a comienzos de año (K) es de 1.000.000 dólares. Si el capital de la compañía se hubiera colocado en una cuenta de ahorros a ese interés del $4 \%$ hubiera obtenido 40.000 dólares de beneficio. Desde el punto de vista clásico consideraríamos que el beneficio total de la compañía tiene dos componentes: 40.000 dólares como interés equivalente y 60.000 dólares como beneficio de empresa. Los economistas neoclásicos camuflan todo ello al considerar el interés equivalente hipotético como un "coste", de la misma naturaleza que los salarios, las materias primas y la depreciación por obsolescencia del capital fijo. Como consecuencia, su definición del beneficio económico es el beneficio de empresa (60.000 dólares). La economía post-keynesiana hace suyos muchos conceptos neoclásicos, entre ellos este. 
La tasa de ganancias es la proporción del beneficio anual al comienzo del año del capital social, es decir, $r=100.000 \$ / 1.000 .000 \$=0.10$. La tasa de beneficio de empresa correspondiente (re) es la suma del beneficio de empresa dividido por el capital social, lo que nos daría re $=60.000 \$ / 1.000 .000 \$$ $=6 \%$. Es fácil ver que la tasa de beneficio de empresa equivale a la diferencia entre la tasa de ganancias y la tasa de interés: $r e=r-i=10 \%-4 \%=6 \%$.

Dos consideraciones adicionales son importantes empíricamente. Primero, el beneficio tal y como es considerado en la contabilidad nacional no es ni el beneficio total $(\mathbf{P})$ ni el beneficio de empresa (PE), sino algo entremedio. Las contabilidades nacionales definen el beneficio económico como el beneficio neto real del interés efectivamente pagado. Por lo que, si la compañía en cuestión ha pedido prestado la mitad de su capital social (500.000 dólares), tendrá que pagar 20.000 dólares en intereses (4\% de su deuda total de 500.000 dólares). De ahí que la contabilidad nacional considere que el beneficio $\left(\mathbf{P}^{\wedge}=80.000\right.$ dólares $)$ sea el beneficio real $(\mathbf{P}=100.000$ dólares $)$ menos el interés pagado sobre el principal de la deuda (20.000 dólares). Por lo tanto, para calcular los beneficios reales necesitamos añadir los intereses desembolsados a la cantidad recogida como beneficio en la contabilidad nacional. Solo entonces podemos calcular el nivel y la tasa de beneficio de empresa de la manera anteriormente descrita (12).

Segundo, es importante señalar que todas las tasas de ganancias sean tasas reales, es decir, ajustadas a la inflación, si usamos beneficios en dólares corrientes en el nominador y el coste real del capital social en el denominador. De esta manera, tanto el numerador como el denominador reflejan el mismo conjunto de precios, que es la base de cualquier calculo real (13). Es obvio en el caso de la tasa de ganancias ( $\mathbf{r}$ ) cuando tanto $\mathbf{P}$ como $\mathbf{K}$ reflejan los precios corrientes. Pero también se aplica a la tasa de beneficio de empresa (re), cuyo nominador es el beneficio corriente adicional al interés equivalente corriente al comienzo del año del coste del capital social corriente ( $\mathbf{P}-\mathbf{i K}$ ). Calculado de esta manera, la tasa de beneficio de empresa $\mathbf{r e}=\mathbf{r}-\mathbf{i}$ es una tasa real. En el Apéndice sobre Fuentes y Métodos pueden encontrarse otros detalles, consideraciones y derivaciones de la manera específica en la que la contabilidad nacional calcula tanto el beneficio como el capital.

Tras haber aclarado este punto, volvemos al análisis de los acontecimientos que han provocado la crisis actual. Primero y ante todo, los movimientos de la tasa de ganancias. 


\section{Las tendencias de post-guerra de la acumulación en EE. UU.}

\section{La tasa de ganancias general}

El Cuadro 1 muestra la tasa de ganancias de las corporaciones no financieras de EE UU, que es la proporción de sus beneficios antes del pago de intereses e impuestos sobre el beneficio al comienzo del año del coste real de sus plantas y equipos. También muestra la tendencia de la tasa de ganancias (ver el Apéndice para los detalles). Como se ha explicado antes, necesitamos calcular los beneficios antes del pago de intereses, porque a continuación compararemos esta cantidad con el interés equivalente del mismo capital social para deducir los beneficios de empresa. Como los beneficios publicados de las corporaciones no financieras no incluyen el pago de intereses netos, añadimos esta cantidad a los beneficios publicados. Este cálculo agregado del beneficio de las corporaciones no financieras incluye una parte de los beneficios de las corporaciones financieras, porque estas últimas obtienen sus beneficios del pago de intereses.

\section{Cuadro 1}

Tasa de ganancias de las corporaciones no financieras de EE.UU. (1947-2010)

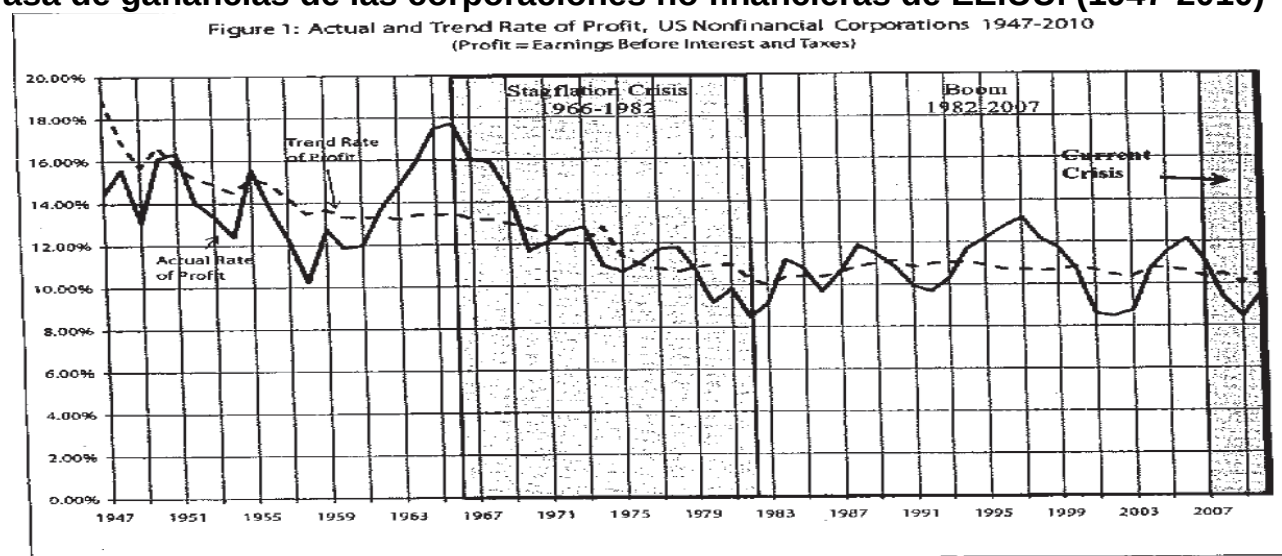

$r=P / K(-1)$, y la tendencia de valor de $r$ (rtendencia)

$P$ es la suma de los beneficios de las corporaciones no financieras de la tabla 1.14 de NIPA, línea 27, hasta el primer trimestre de 2010; y los intereses monetarios netos de las corporaciones no financieras de la tabla 7.11, línea 11 menos la línea 17, que solo esta disponible anualmente hasta 2008 y que se proyectó hasta 2010 usando la tendencia de sus componentes individuales. Los beneficios de las corporaciones tan y como los recoge NIPA son netos, sin el pago de los intereses monetarios netos, por lo que necesitamos sumar estos para calcular los beneficios antes del pago de intereses. Ello nos da el equivalente del NIPA del calculo del habitual de la contabilidad empresarial "Ganancias antes de intereses e impuestos" (EBIT). Este paso es necesario porque a continuación restaremos el interés equivalente a todo el capital (no solo el interés neto pagado del capital crediticio) para obtener el total y la tasa de beneficio de empresa (ver 
los cálculos para el Cuadro 6 más adelante).

El denominador de la tasa de ganancias es el capital avanzado ese año. Ya que NIPA recoge el capital social a fin de año, es necesario usar el coste real del capital social de las corporaciones no financieras del año previo $(K(-1))$. El capital social a fin de año aparece en FA en la tabla 6.1, linea 4. Los datos de FA se facilitan anualmente hasta 2008, con una proyección tendencial para 2009.

La rtendencia ha sido calculada utilizando una regresión LOESS en Eviews 5 con $P$ y $K$ $(-1)$ con un ancho de banda $=0.50$. LOESS es el tipo de regresión más cercana con una polynomia de grado 1 (linear) y un peso local tricúbico. Esta técnica no es sensible a las fluctuaciones a corto, lo que la hace util para la estimación de tendencias. Rtendencia fue generada dividiendo el valor de la tendencia de $P$ por $K(-1)$.

Observamos que la tasa de ganancias real esta sujeta a muchas fluctuaciones y puede verse muy influenciada a corto por acontecimientos históricos. Por ejemplo, el gran crecimiento de la tasa de ganancias de los años 1960 refleja la escalada militar paralela de la Guerra de Vietnam. Las guerras son en general buenas para los beneficios, al menos en sus inicios. La tendencia de la tasa de ganancias que aparece en una línea discontinua en el Cuadro 1 tiene como objetivo diferenciar las causas estructurales de la tendencia de la tasa de ganancias de las fluctuaciones a corto, resultado de los acontecimientos coyunturales como la Guerra de Vietnam. Vemos que la tendencia de la tasa de ganancias cae durante treinta años y se estabiliza después. La pregunta es: ¿qué ocurrió para cambiar esta tendencia?

\section{Productividad y salarios reales}

El Cuadro 2 nos da la clave. Refleja las relaciones entre la productividad horaria y la compensación real por hora (salarios reales) en el sector empresarial de EE UU de 1947 a 2008. Los salarios reales tendieron a crecer más despacio que la productividad, es decir, la tasa de explotación tendió a crecer. Pero al comienzo de la Administración Reagan, en los años 1980, el crecimiento de los salarios reales se freno considerablemente. Basta con comparar los salarios reales desde 1980 con la tendencia que hubieran seguido si se hubieran mantenido su relación con la productividad de post-guerra. Esta diferencia de tendencias fue el resultado de ataques concertados contra los sindicatos en ese periodo. Veremos que el impacto en la tasa de ganancias fue dramático, porque la compensación salarial es un componente relativo importante de la tasa de beneficio. 


\section{Cuadro 2}

La productividad horaria del sector empresarial y la compensación horaria real e hipotética

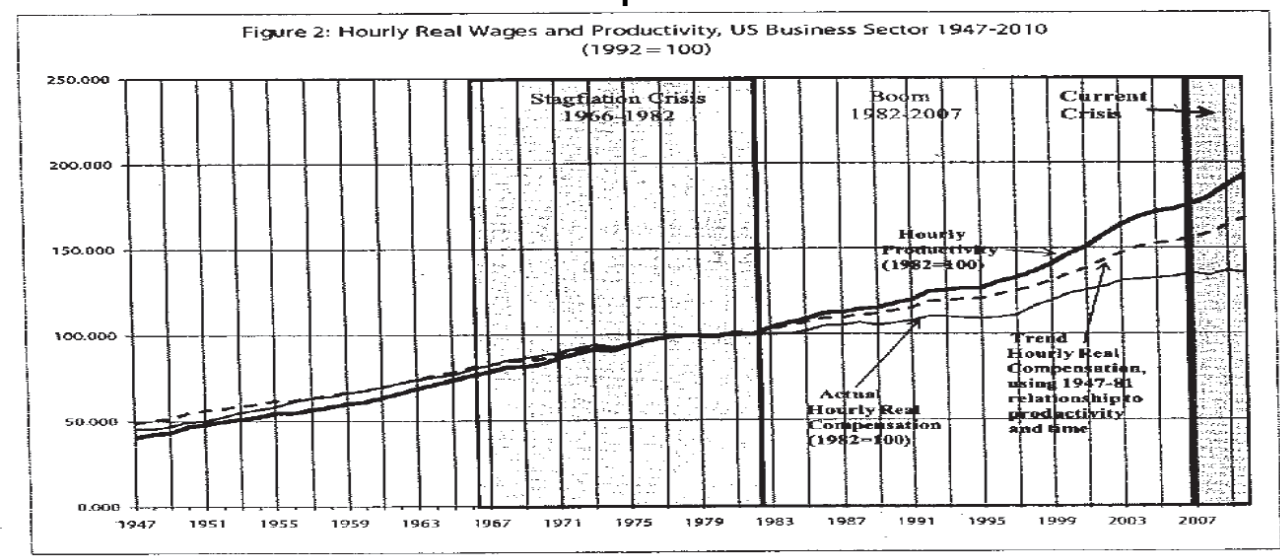

La productividad horaria y la compensación real están disponibles en el US Bureau of Labor Statistics (BLS), en el apartado "Major Sector Productivity and Costs Indexes", en www.bls.org. Las cifras de 2010 son para el primer trimestre. La relación entre productividad (y) y compensación real al trabajador (ec) mantienen una tendencia continua en la "Edad de Oro" de post-guerra de 1960-1981, que se obtuvo con una regresión de $\ln (e c)$ sobre $\ln (y)$ y una tendencia temporal (aunque no fue significativa). La tendencia fue después proyectada para el periodo 1982-2009 para calcular la hipotética curva que (ec) hubiera seguido si se hubiera mantenido la tendencia anterior (ecc). Si se utiliza los datos de 1960-1981, la tendencia hipotética es más modesta que la resultante de los datos de 1947-1981. He utilizado la opción más modesta para evitar sobredimensionar el efecto benéfico en la tasa de ganancias de la disminución del crecimiento de los salarios reales en el período Reagan-Thatcher.

\section{El impacto en los beneficios de la supresión de los salarios rea- les}

El Cuadro 3 muestra el profundo impacto que el freno del crecimiento de los salarios reales tuvo en los beneficios. Refleja tanto la tasa de ganancias real como la tendencia hipotética que hubiera seguido si los salarios reales en las corporaciones no financieras hubieran mantenido la relación de post-guerra con la productividad de dicho sector. La represión contra los trabajadores y los sindicatos que se inició en la época de la Administración Reagan tenía un objetivo claro: alimentar el boom de finales del Siglo XX. 


\section{Cuadro 3}

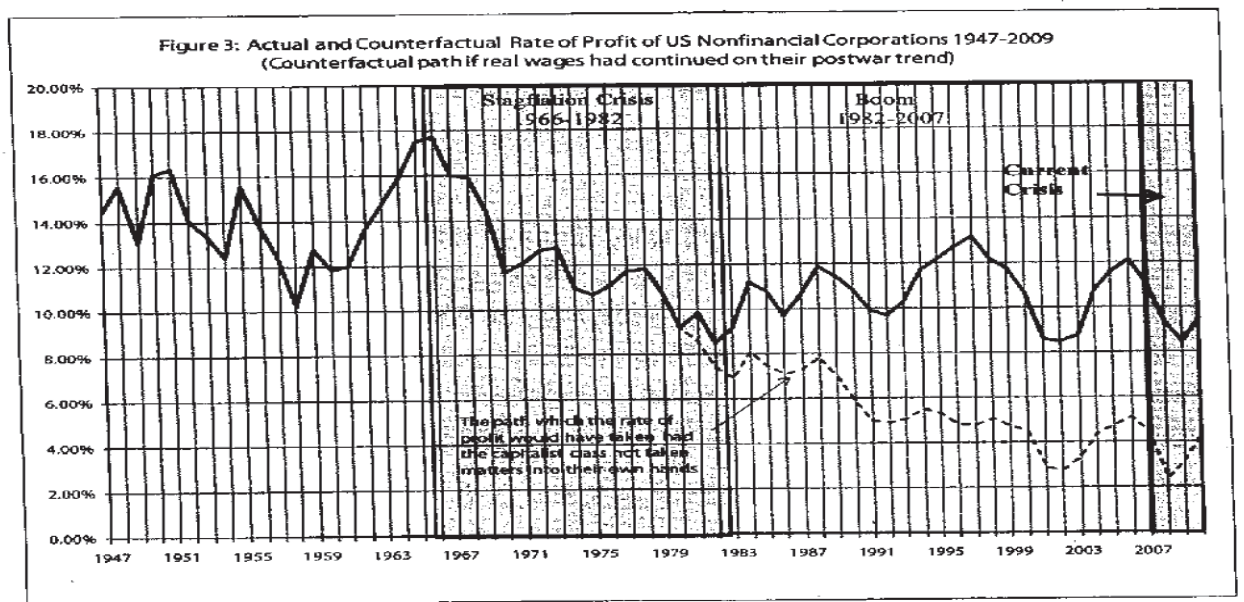

La tasa de ganancias real ( $r$ ) comparada con la tasa de ganancias hipotética $(r c)$.

Se han usado las variables previamente calculadas para crear una relación entre la compensación horaria al trabajador hipotética y la compensación horaria real ( $z=$ ecc/ec). Desde 1982, la compensación real horaria total de las corporaciones no financieras $(E C)$ se multiplicó por $z$ para estimar la compensación total que habrían recibido los trabajadores $(E C C C)$ si los salarios hubieran mantenido la tendencia pre-1982. La diferencia entre $(E C C-E C)$ representa el beneficio obtenido gracias a una ralentización del salario real. Al sumarlo a los beneficios reales nos proporciona los beneficios hipotéticos, y al dividir los beneficios hipotéticos por el capital social $\mathrm{K}(-1)$ nos proporciona una estimación de la tasa de ganancias hipotética.

\section{La extraordinaria caída de la tasa de ganancias}

Acabamos de ver como la caída en la tasa de ganancias fue frenada a través de una reducción sin precedentes del crecimiento de los salarios reales. Pero solo explica parcialmente el gran boom que comenzó en los años 1980. Al comienzo de este artículo subrayé que lo que impulsa la acumulación capitalista es la diferencia entre la tasa de ganancias y la tasa de interés, es decir, la tasa de beneficio de empresa. Y es ahí precisamente donde se haya la otra clave del gran boom: la extraordinaria y sostenida reducción de la tasa de interés que se inició más o menos al mismo tiempo. El Cuadro 4 refleja la tasa de interés a tres meses de los bonos del Tesoro en EE UU, así como el índice de precios de los bienes de capital (pk), que aparecen representados por una línea discontinua. En la primera fase, de 1947 a 1981, la tasa de interés creció 24 veces, del 0,59\% en 1947 al 14,03\% en 1981. En la segunda fase, de 1981 en adelante, cayó también dramáticamente, del 14,03\% a un mero $0,16 \%$ en el 2009. Para poder distinguir la influencia del mercado de las intervenciones regulatorias sería necesario discutir la teoría de la tasa de interés determinada competitivamente, lo que no es posible en las dimensiones de este artículo (15). En cualquier caso, sea el que sea el peso relativo de los 
factores del mercado y de las intervenciones regulatorias administrativas, el largo ascenso y consecuente laga caída de la tasa de interés fue evidente en la mayor parte de los países capitalistas. El Cuadro 5 es una muestra de ello, al comparar la tasa de interés en EE UU con la media de sus principales socios comerciales. Entre otras cosas, ello demuestra que la dinámica que observamos en EE UU era una característica compartida por el centro del capitalismo en su conjunto.

Cuadro 4

La tasa de interés y el nivel de los precios

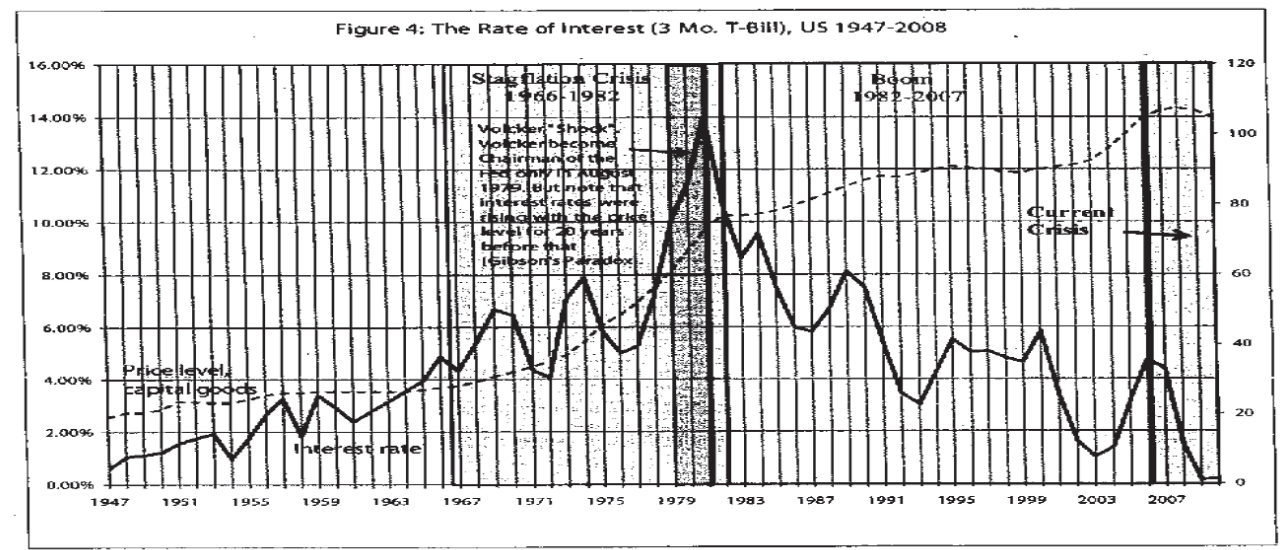

La tasa de interés es la tasa del bono del Tesoro a tres meses, que se puede encontrar en la tabla 73, primera columna en The Economic Report of the President publicado por el BEA en www.gpoaccess.gov/eop/tables10.html. Los niveles de precios utilizados son los de los bienes de capital nuevos, ya que es el indicador relevante de la capacidad de compra del beneficio. Se pueden obtener en NIPA, tabla 1.1.9, línea 7 (fixed investment deflator).

\section{Cuadro 5}

Las tasas de interés de los EE.UU. Y sus socios comerciales

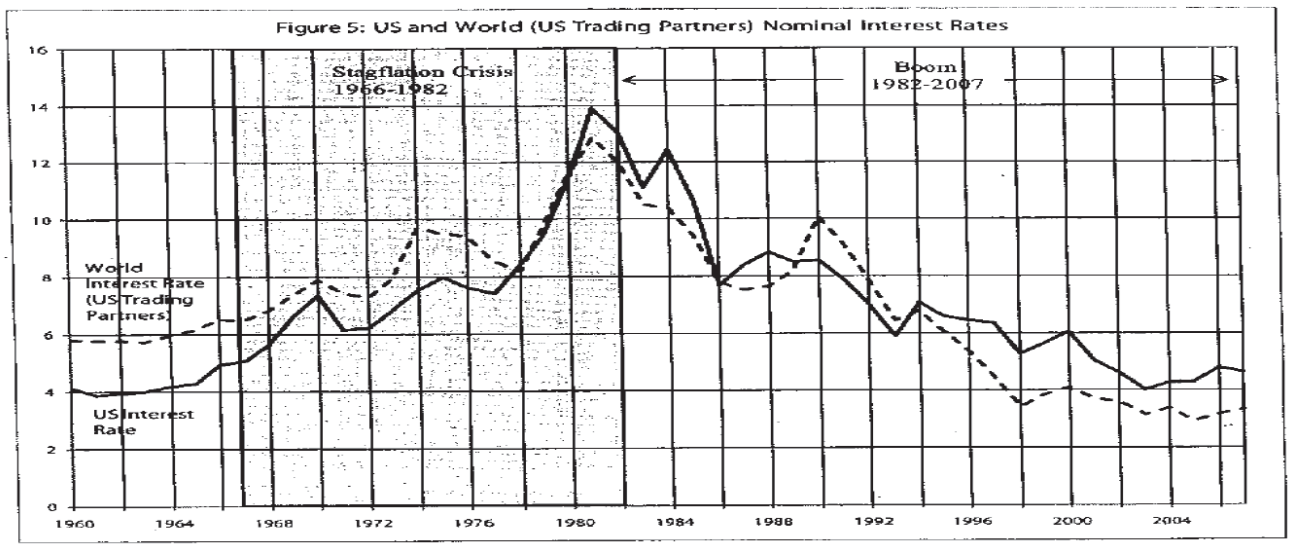

Ya se han descrito las tasas de interés de EE UU. Las de sus socios comerciales se han obtenido de la "Federal Reserve Board Indexes of the Foreign Exchange Value of the Do- 
llar" ( www.federalreserve.gov/releases/h10/Weights). Se han utilizado para deducir una tasa de interés media ajustada de las Estadísticas Financieras Internacionales (IFS) del Fondo Monetario Internacional (FMI). Estoy muy agradecido a Amr Ragab por estos cálculos.

\section{La tasa de beneficio de empresa y el gran boom después de los años 1980}

Podemos ahora juntar todos estos elementos. La diferencia entre la tasa de ganancias general (calculo en bruto de los intereses monetarios netos pagados) y la tasa de interés es la tasa de beneficio de empresa. Este es el motor central de la acumulación, la base material de los "espíritus animales" del capitalismo industrial. El Cuadro 3 muestra que la tasa de ganancias general fue resucitada de su largo declive con un ataque concertado contra los trabajadores que hizo que los salarios reales después de 1982 crecieran mucho más despacio que en el pasado. Los Cuadros 4 y 5 reflejan que la tasa de interés cayó radicalmente después de 1982. El Cuadro 6 demuestra que el efecto neto de estos dos movimientos sin precedentes históricos fue elevar de manera muy significativa la tasa de beneficio de empresa. Este es todo el secreto del gran boom que comenzó en los años 1980.

El gran boom era inherentemente contradictorio. La caída dramática de la tasa de interés desató una fiebre crediticia, y el peso de la deuda sectorial creció también dramáticamente. Los hogares, cuyos ingresos reales se habían reducido por la contracción del crecimiento de los salarios reales, fueron tentados con créditos cada vez más baratos para poder mantener el crecimiento del consumo. En consecuencia, como se muestra en el Cuadro 7, la relación entre deuda e ingresos de los hogares creció de manera desorbitada en los años 1980. En segundo lugar, una vez que la tasa de interés cayó hasta cero (actualmente se sitúa en el 0,0017, es decir, el 0,17\%), no se puede seguir rebajándola. Aunque es cierto que la diferencia entre la tasa base y la tasa de interés a la que se presta a las empresas y a los consumidores (créditos al consumo, personales, hipotecarios...) puede aun regularse a la baja por el estado. Pero la diferencia entre la tasa base y el resto de las tasas comerciales de interés es la fuente de beneficios del sector financiero, que recibe dinero a una tasa base de interés y lo presta a otras. Las posibilidades de reducir la diferencia entre una y otras es, por lo tanto, limitada. 


\section{Cuadro 6}

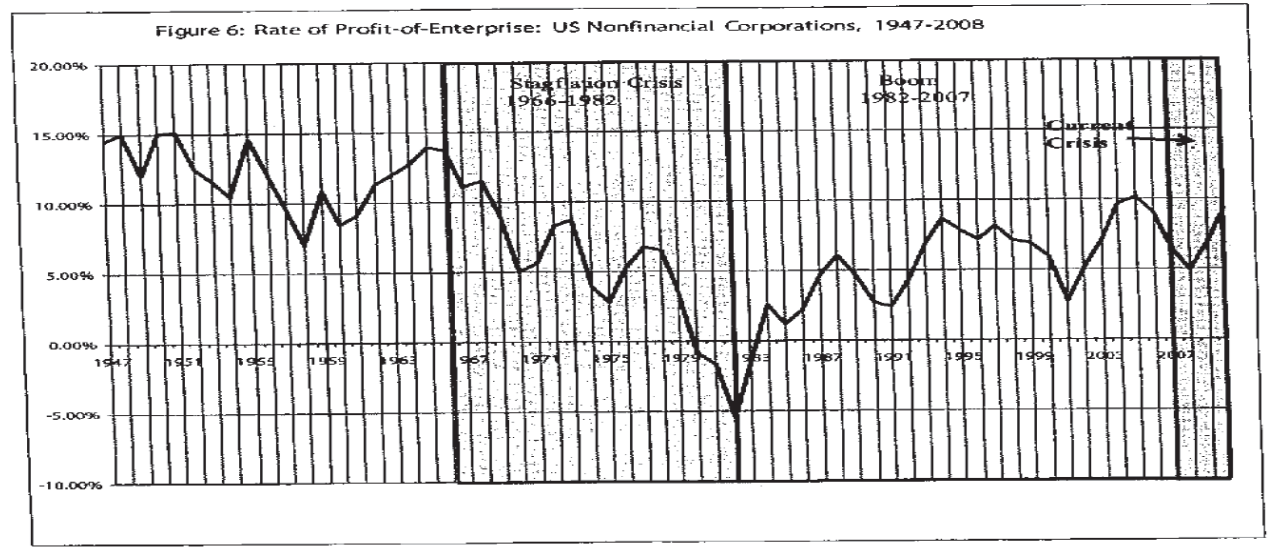

$r e=r-i$, donde $r$ e $i$ han sido ya explicados.

Pero ¿qué ocurre si la relación entre deuda e ingresos crece? Después de todo, si la deuda es más barata, es posible endeudarse más sin incurrir en un servicio de la deuda mayor (la relación entre la amortización y el pago de los intereses con los ingresos).

\section{Cuadro 7}

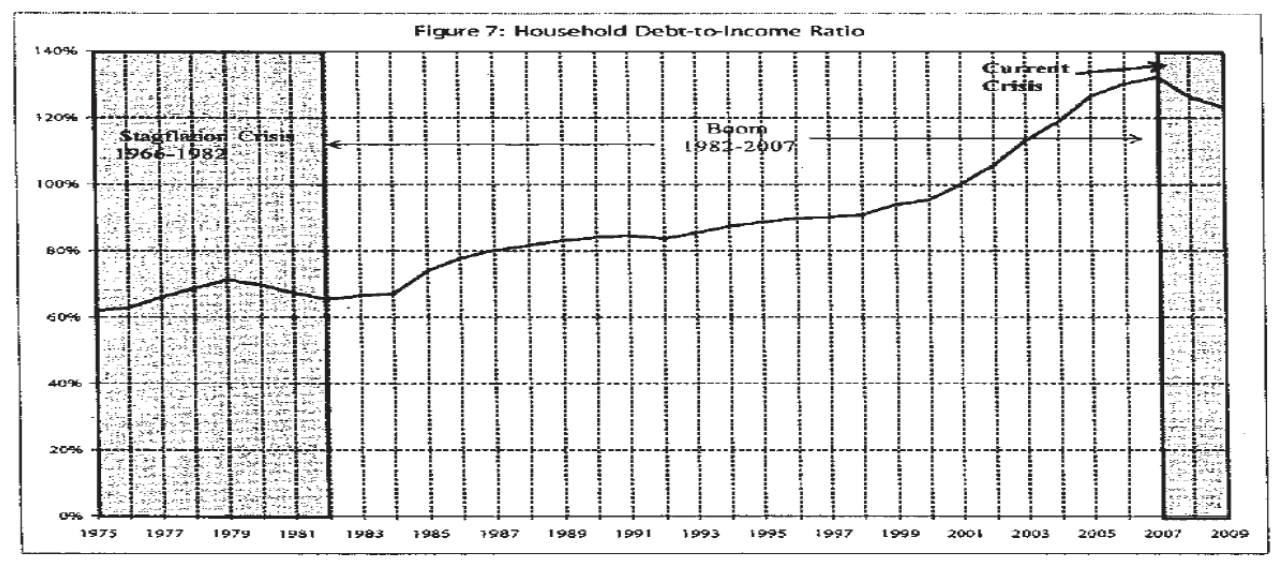

Relación entre la deuda de los hogares con el ingreso personal disponible. El anterior se obtiene de la tabla D3, línea 2 del "Federal Reserve Bank's Flow of Funds", y la primera de NIPA, tabla 2.1, línea 26. 
Y, de hecho, como se ve en el Cuadro 8, mientras que la relación entre deuda e ingreso creció de manera sostenida en los años 1980, la correspondiente relación entre deuda y servicio de la deuda se mantuvo en unos márgenes estrechos: los hogares se endeudaban más, pero sus pagos mensuales no aumentaban mucho. Pero en los años 1990, como la deuda continuó ampliándose, el servicio de la deuda también comenzó a crecer. Hacia 2007, la deuda alcanzó el punto histórico más alto de su curva, para caer a continuación en 2008 en la medida que la deuda caía más deprisa que los salarios en el torbellino de la crisis.

Lo que hace destacar un punto importante. Del lado de los trabajadores, el declive de la tasa de interés promovió el aumento de la deuda de los hogares, lo que ayudo temporalmente a mantener su nivel de vida a pesar de la reducción del crecimiento de los salarios reales. Desde un punto de vista macroeconómico, el crecimiento resultante del gasto de los hogares alentó el boom. El principal ímpetu del boom era la caída dramática de la tasa de interés y la igualmente dramática caída de los salarios reales en relación con la productividad (el aumento de la tasa de explotación), que en conjunto elevaron significativamente la tasa de beneficio de empresa. Las dos mismas variables jugaron papeles diferentes en distintos sitios. Pero los dados estaban cargados.

\section{Las lecciones de la Gran Depresión de los años 1930}

En la medida en que la crisis actual se ha agudizado, los gobiernos de todo el mundo se han movilizado para salvar a bancos y empresas de la quiebra, creando con frecuencia de manera discontinua grandes cantidades de dinero para ello. Todos los países avanzados cuentan con los llamados estabilizadores automáticos, como las subvenciones de paro y ayudas sociales, que crecen en una fase depresiva. Pero estos estabilizadores automáticos están concebidos para una recesión, no para una depresión. Los gobiernos han sido mucho menos entusiastas en la creación de nuevas formas de gasto público para ayudar directamente a los trabajadores. De hecho, incluso en lo que se refiere a la cuestión del déficit fiscal hay una fuerte división en dos campos sobre la política económica a seguir. 


\section{Cuadro 8}

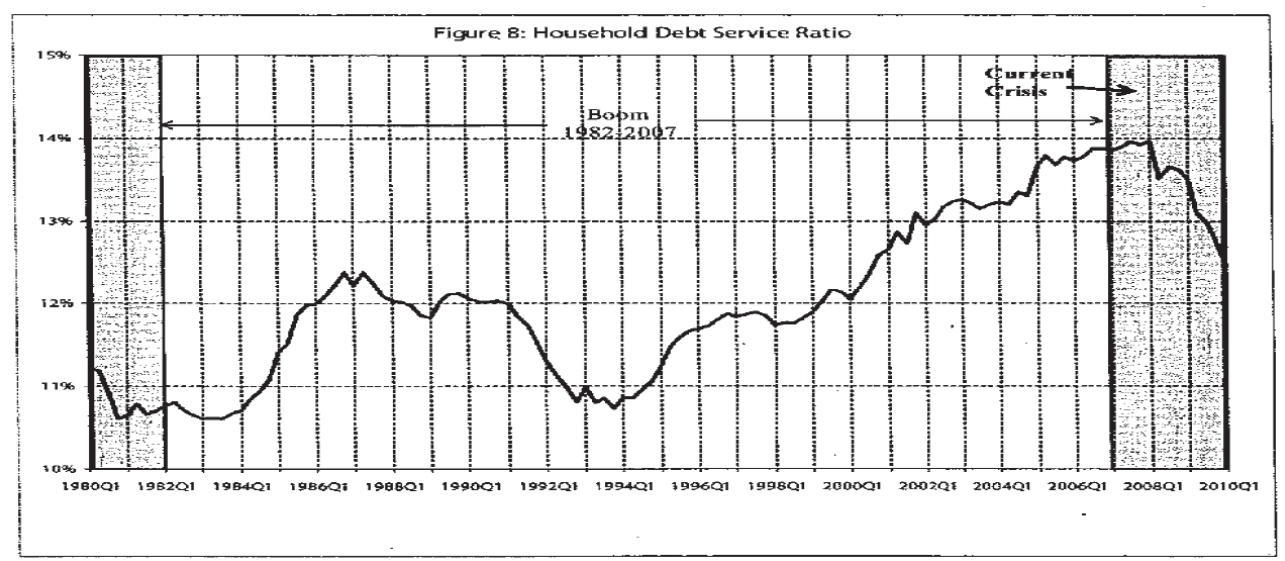

Relación entre el servicio de la deuda (pago de amortizaciones e intereses de las hipotecas activas y los créditos al consumo) con el ingreso personal disponible, que aparece como la variable DSR en la tabla "Flow of Funds" que lleva por título "Household Debt Service and Financial Obligations Ratios", que se puede consultar en www.federalreserve.gov/releases/housedebt/dafault.htm.

Esta división fue patente en el último G-20 de Toronto, en junio del 2010. De un lado, la ortodoxia, con su defensa de la "austeridad", concepto que en realidad quiere decir recortes en el gasto público en sanidad, educación, seguridad social y otras partidas que benefician especialmente a los trabajadores. Jean-Claude Trichet, Presidente del Banco Central Europeo, afirmó en la reunión que "la idea de que las medidas de austeridad pueden provocar un estancamiento económico es incorrecta". "Los Gobiernos no deben caer en la adicción de endeudarse como una solución rápida para estimular la demanda....el déficit fiscal no puede convertirse en una situación permanente", añadió el Ministro de Finanzas alemán, Wolfang Schauble. En parte estas declaraciones surgen de una fe de carbonero en la visión de la economía ortodoxa de que los mercados son casi perfectos y se recobran rápidamente por si solos. Después de todo, la tasa de beneficio de empresa de las corporaciones no financieras que muestra el Cuadro 6 señala una recuperación significativa en 2010. Y para algunos bancos de inversión, el dinero ha sido como petróleo en el Golfo de México: bastaba con recogerlo de la superficie del mar. En 2010, los beneficios de Goldam Sachs en el primer trimestre fueron 3.300 millones de dólares, el doble que el año anterior, y siendo el segundo trimestre de más beneficios desde que en 1999 cotiza en bolsa. Desde el optimismo de la teoría ortodoxa, ello parece sugerir que la felicidad esta de nuevo a la vuelta de la esquina. Los banqueros centrales europeos aun recuerdan como una pesadilla la hiperinflación alemana de los años 1920 como consecuencia del déficit fiscal y sus devastadoras consecuencias tanto políti- 
cas como sociales. Finalmente, queda la cuestión práctica de los potenciales beneficios para el capital europeo de los programas de austeridad. El movimiento obrero europeo sobrevivió la era neoliberal en mejores condiciones que el británico o el norteamericano y, como demostraron Reagan y Tatcher, una crisis es la excusa perfecta para atacarlo. Desde este punto de vista, la posibilidad de que la austeridad ponga las cosas mucho peor para el conjunto de la población es un riesgo aceptado si debilita a la vez la resistencia de la fuerza de trabajo.

La delegación de EE UU en la reunión del G-20 defendió una posición distinta. Sólo en los EE UU la riqueza de los hogares ha menguado en billones de dólares y la venta de casas nuevas esta por debajo de los niveles de 1981. Más aun, la Organización Internacional del Trabajo ha advertido recientemente que estamos ante una "prolongada y severa" crisis global de empleos. Algo que una potencia imperial tiene que tomar muy en serio, sobre todo cuando esta implicada en múltiples guerras y "acciones de policía" globales. Finalmente, aquí también hay un aspecto histórico esencial. El Presidente Obama urgió a los líderes europeos a repensar su posición, señalando que debían "aprender de los errores fundamentales del pasado, cuando se retiraron los estímulos demasiado pronto y el resultado fue nuevas dificultados económicas y recesión" (16). Los "errores fundamentales del pasado" a los que se refiere Obama tienen que ver con los acontecimientos de los años 1930. La Gran Depresión iniciada por la colapso de la bolsa en 1929 provocó una caída profunda de la producción y un grave crecimiento del paro de 1929 a 1932. Pero en los siguientes cuatro años, la producción volvió a crecer casi un $50 \%$, mientras que las tasas de paro se redujeron un tercio y el gasto público creció casi un $40 \%$. De hecho hacia 1936, la producción estaba creciendo a un extraordinario $13 \%$. La contrapartida fue que el presupuesto federal alcanzó un déficit de casi el $5 \%$ en esos mismos cuatro años. Así que en 1937 la Administración Roosevelt aumento los impuestos y redujo drásticamente el gasto público (17). El PIB real cayó rápidamente y el paro volvió a crecer de nuevo. Reconociendo su error, el Gobierno rápidamente corrigió sus políticas y volvió a aumentar sustancialmente el gasto y el déficit públicos en 1938. En 1939 la producción volvió a crecer un 8\%. Solo entonces EE UU comenzó a prepararse para una posible guerra y solo en 1942 fue esta la prioridad económica. El Cuadro 9 muestra el crecimiento del PIB durante estos años críticos.

Unas cuantas lecciones pueden aprenderse de todo ello. Primero, la reducción del gasto público durante una crisis es un "error fundamental". Al menos desde el punto de vista de Obama. Segundo, es obvio que la economía comenzó a recuperarse en 1933 y que, con la excepción del error de la Administración Roosevelt de reducir el gasto público en 1937, siguió recuperándose hasta la preparación para la guerra de EE UU en 1939 y su entrada en la misma en 1942 (Pearl Harbour tuvo lugar el 7 de diciembre de 1941). Es por 
lo tanto un error atribuir la recuperación, que comenzó nueve años antes de la guerra, a esta. La guerra estimuló aun más la producción y el empleo. Tercero, es sin embargo correcto afirmar que el gasto público en tiempo de paz jugó un papel crucial a la hora de acelerar la recuperación. Cuarto, el gasto público implicado no solo fue utilizado para la compra de bienes y servicios. También se dedicó a la contratación de empleo público para la realización de infraestructuras y servicios públicos. Por ejemplo, la Administración de Trabajos Públicos (WPA) llegó a emplear millones de personas en la construcción pública, en las artes, en la enseñanza y en los servicios sociales para los pobres.

\section{Cuadro 9}

Crecimiento real del PIB durante la Gran Depresión (1929-1942)

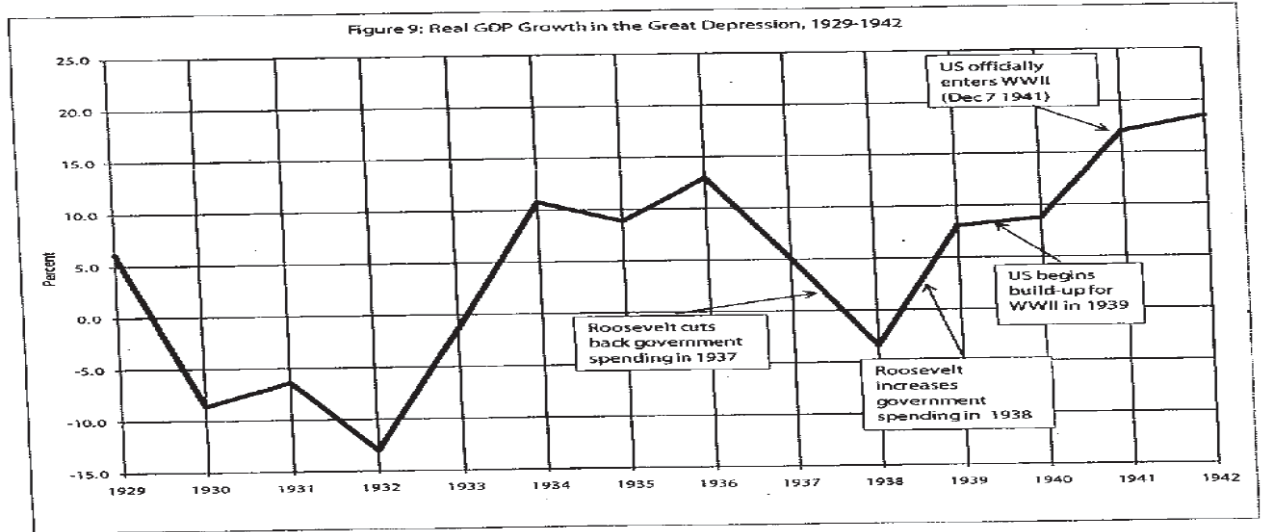

El crecimiento real del PIB se puede obtener desde 1930 en adelante en la tabla 1.1.1, línea 1 de NIPA. La tasa decrecimiento de 1929 fue calculada usando datos de 1928-29 (749,700, 843, 334) de The World Economy: Historical Statistics, de la OCDE, Paris 2003.

\section{Algunas implicaciones políticas para el presente período}

El gasto público puede estimular mucho una economía. Es evidente en períodos de guerra, que en general están acompañados de un gasto público financiado por un déficit fiscal masivo. En la II Guerra Mundial, por ejemplo, en 1943-45 el déficit presupuestario de EE UU alcanzó el 25\%. Hoy, en contraste, el déficit presupuestario en el segundo trimestre de 2010 es menos del $11 \%$. En cualquier caso, es importante señalar que una guerra es una forma particular de movilización social que sirve para aumentar la producción y el empleo. En esos casos, una parte de la creación de empleo resultante es debido a la demanda de armamento y de otros bienes y servicios esenciales para la guerra así como la demanda que a su vez generan. Pero otra parte 
es debido al empleo directo por parte del ejército, la administración gubernamental, la seguridad, el mantenimiento y reparación de infraestructuras públicas y privadas etc... Por lo tanto, incluso en una guerra tenemos que diferenciar entre dos formas diferentes de estímulo económico: la demanda directa gubernamental que estimula el empleo siempre y cuando las empresas no se queden con la mayor parte del dinero o lo utilicen para pagar sus deudas; y el empleo público directo, que a su vez estimula la demanda siempre y cuando las personas así empleadas no ahorren sus salarios o lo usen para pagar sus deudas.

Estas dos formas de estímulo pueden utilizarse también en tiempo de paz para el gasto público en una movilización social para hacer frente a la crisis. En la primera modalidad, el gasto público se dirige a las empresas y los bancos, con la esperanza de que lo utilicen para la creación de empleo. Es la formula tradicional keynesiana: estimular el sector privado y confiar que el aumento de beneficios cree empleo. En la segunda modalidad, el gobierno utiliza el gasto publico para contratar directamente a aquellos que no encuentran trabajo en el sector privado y, en la medida que estos nuevos asalariados gastan sus ingresos, los beneficios acaban afectando positivamente a las empresas y los bancos. La cuestión de que efectivamente se vuelvan a gastar los dineros recibidos del gobierno es esencial. Los bancos y las empresas no financieras han recibido en los últimos años grandes cantidades de dinero para "sanearlas" en los principales países del mundo. Pero en la mayoría de los casos estas grandes cantidades de dinero han acabado en sus cajas fuertes: los bancos las necesitan para cubrir sus activos "enfermos" y las industrias para pagar sus deudas. Como es lógico, ninguno de ellos considera sensato invertir o utilizar este dinero en unas circunstancias en las que hay pocas esperanzas de obtener un beneficio conveniente. Por lo tanto, muy poco de la gran masa de dinero utilizada para "sanear" a los bancos y las empresas ha terminado en forma de salarios. Pero si se utilizara la segunda modalidad, la cosa sería muy diferente. Los ingresos de quienes fueran empleados tendrían que ser gastado inevitablemente, porque tienen que consumir para vivir. La segunda modalidad tiene por lo tanto dos grandes ventajas: crearía empleo directamente para aquellos que más lo necesitan; y generaría un tirón de la demanda que beneficiaria a las empresas que produjeran para ellos.

¿Qué impide entonces a los gobiernos que creen programas de empleo público directo? La respuesta, por supuesto, es que el capital prefiere el estimulo directo a las empresas. De hecho, como el empleo directo público subordina la búsqueda de beneficios al bien social, es visto correctamente como una amenaza al orden capitalista y como "socialista". Más aún, interferiría con los planes neoliberales de utilizar fuerza de trabajo barata internacionalmente, lo que permite no solo un coste de producción más barato en terceros países sino también frena el crecimiento de los salarios en las metrópolis. La cues- 
tión clave en nuestra época es por lo tanto si podemos lograr una movilización social lo suficientemente potente para hacer frente a la Gran depresión sin tener que recurrir a la guerra. Es una cuestión global, porque el paro, la pobreza y la degradación medioambiental son globales. Pero la movilización, por su propia naturaleza, es local. El objetivo es lograr dicha movilización social y extenderla, contra la resistencia de poderosos intereses y estados cobardes.

\section{Notas}

(1) La crisis de 1825 es considerada la primera crisis industrial real. La crisis de 1847 fue tan dura que provocó una oleada revolucionaria en toda Europa. Ver Maurice Flamant y Jeanne Singer-Kerel, Modern Economic Crises, London: Barrie and Jenkins, 1970, pp. 16-23. El termino "la larga depresión de 1873-1893" es de Forrest Capie y Geoffrey Word, "Great Depressions of 1873-1896", en D. Glasner y T. F. Cooley, ed. Business Cycles and Depressions: An Enciclopedia, New York: Garland Publishing, 1997. La Gran Depresión de 1929-1939 es bien conocida. La cronologia de la Gran Stagflacion de 1967-1982 es de Shaikh, "The Falling Rate of Profit and the Economic Crises in the US" en R. Cherry et al., ed., The Emperiled Economy, New York: Union for radical Political Economy, 1987. Tanto el nombre como la cronología de la crisis económica internacional que estalló en 2008 están aun en estudio.

(2) John Kenneth Galbraith, The Great Crash, 1929, Boston: Houghton Miflin, 1955, cap. I-II y pp. 182, 192. Galbraith era ambivalente sobre la posibilidad de que se repitiera una Gran depresión. Como historiador era muy consciente de que los "ciclos financieros de euforía y pánico...concuerdan mas o menos con tiempo que tardó la gente en olvidarse del último desastre". John Kenneth Galbraith, Money: Whence it Came, Where it Went, Boston: Houghton Miflin, 1975, p. 21. Advirtió que estos ciclos son "el resultado de la libre decisión y opción de cientos de miles de individuos", que a pesar de la esperanza de que memoria del ultimo acontecimiento halla tenido efectos inmunizadores "las posibilidades de que se vuelva a producir una orgía especulativa son bastante altas", que "durante el próximo boom se volverá a redescubrir las virtudes del sistema de libre empresa", que entre "los primeros en aceptar estos argumentos estarán algunos que fueron responsables en invocar la necesidad de controles...y que entonces nos dirán con toda seguridad que son innecesarios", y que con el tiempo "las instituciones reguladoras se convertirán, con algunas excepciones, bien en parte de la propia industria 
que pretenden regular o en algo obsoleto". Galbraith, The Great Crash, 1929, pp. 4-5, 171, 195-96. A pesar de todo, en tanto que político y funcionario continuó confiando en que ninguno de aquellos acontecimientos volviera a repetirse.

(3) Floyd Norris, "Securization Went Awry Once Before", New York Times, 29 de enero de 2010.

(4) David Smith, "When Catastrophe Strikes Blame a Black Swan", The Sunsay Times, 6 de mayo de 2007.

(5) Shaikh, "The Falling Rate of Profit"; J.J. van Duijn, The Long Wave in Economic Life, London: Allen and Unwin, 1983, cap. 1-2.

(6) E. Mandel, Late Capitalism, London: New Left Books, 1975, pp. 126-127.

(7) Shaikh, "The falling Rate of Profit", p.123.

(8) John Maynard Keynes, A Treatise on Money, New York: Hartcourt, Brace and Company, 1976, p. 148.

(9) Lewis Braham, "The Business Week 50", Business Week, 23 de marzo de 2001.

(10) Donald Rumsfeld, "DoD New Briefing -Secretary Rumsfeld and Gen. Myers, United States Department of defense, 12 de febrero de 2002, en www.defense.gov.

(11) Eckhard Hein, "Money, Credit and the Interest Rate in Marx's Economics: on the Similarities of Marx's Monetary Analysis to Post-Keynesian AnaIysis", International Papers in political Economy, 11 (2), 2004, pp.20-23; Karl marx, Capital, Volume III, New York: International Publishers, 1967, cap. XXIII; Shaikh, "The Falling Rate of Profit", p. 126n1.

(12) He defendido en otro lugar que el cálculo mas apropiado del capital es el del capital social bruto a precios corrientes. Shaikh, "Explaining the Global Economic Crises: A Critique of Brenner", Historical Materialism, 5, 1999, pp. 106-7. Pero este concepto no es recogido por la mayor parte de las contabilidades nacionales, porque han adoptado el criterio de que los bienes de capital se deprecian geométricamente sobre un periodo de tiempo infinito. Esta 
concepción "es utilizada ampliamente en las exposiciones teóricas de la teoría neoclásica del capital dada su simplicidad", a pesar del hecho de que muchos la consideren "empíricamente imposible". Charles R. Hulten, "The Measurement of Capital", en E. R. Bernt and J. E. Triplett, eds, Fifty Years of Economic Measurement: The Jubilee of the Conference of Research on Income and Wealth, Chicago: University of Chicago Press, 1990, p. 125 . El "resto infinito" que asume también causa muchos problemas. Michael J. Harper, "The Measurement of Productive Capital Stock, Capital Wealth, and capital Services", BLS Working Paper N 128, US Bureau of Labor Statistics, 1982, pp. 10, 30. La asunción de una vida infinita hace imposible el cálculo del capital social bruto porque depende del tiempo de vida útil real de cada uno de los bienes de equipo. En un futuro trabajo demostraré como se puede calcular el capital social bruto combinando información disponible sobre la vida útil de bienes de equipo específicos con nuevas reglas deducidas para la conducta de los capitales sociales agregados, en un ajuste en cadena. Este nuevo calculo del capital social cambia las tendencias observadas de la tasa de ganancias de 1947-1982, pero tiene solo un impacto limitado en las tendencias a partir de 1982, que son el objetivo de este artículo.

(13) La tasa de ganancias es por definición una relación entre magnitudes monetarias. Por lo tanto, podemos escribirla como $r=P / K$ en la que tanto el beneficio $P$ como el capital $K$ están calculados en precios corrientes. De manera alternativa, podemos deflacionar el denominador por el índice de precios del capital $P k$ para convertir $K$ a coste corriente en $K r{ }^{\circ} K / P K$, el verdadero capital social ajustado a la inflación. Para preservar la homogeneidad dimensional en la relación debemos también deflacionar el numerador por $P k$ para convertir el beneficio nominal $P$ en $P r^{\circ} P / P k$, la verdadera masa de beneficios medida en términos de su poder de compra en relación con el capital. La relación entre ambas mediciones reales es de nuevo $r$.

(14) Al calcular la tasa de beneficio de empresa no hacemos asunción alguna sobre la determinación de la tasa de interés nominal. La habitual hipótesis neoclásica de Fisher es que la tasa de interés real (ir) se define como la diferencia entre la tasa de interés nominal (i) y una tasa de inflación hipotética asumida por el inversor medio $(P e)$. Bajo la asunción adicional de que la tasa de interés real viene dada exógenamente, ello implica que la tasa de interés nominal sigue la tasa de inflación prevista. Pero en una previsión racional, la tasa de inflación prevista seguirá la tasa de inflación real. Por lo que el debate se reduce a la hipótesis de que la tasa de interés nominal sigue a la tasa de inflación: una asunción que ha sido tantas veces desmentida que solo sobrevive en los libros de texto. Pierluigi Ciocca y Giangiacomo Nardozzi, The High Price of Money: An Interpretation of World Interest Rates, Oxford: Claredon Press, 1996, p. 34. El descubrimiento opuesto, conocido desde los tiempos de Tooke y Marx, redescubierto por Gibson, y reelaborado por Keynes, es que la tasa de interés sigue en su mayor parte el nivel de precios y no a 
su tasa de cambio. Esta afirmación ha sido tan desconcertante para la economía ortodoxa que ha sido embalsamada bajo la denominación de "paradoja de Gibson". J. Huston McCulloch, Money and Inflation: A Monetarist Approach, New York: Academia Press, 1982, pp. 47-49.

(15) Para poder medir en que manera los extraordinarios movimientos de la tasa de interés fueron provocados por decisiones de política económica, sería necesario desarrollar una teoría adecuada de las determinantes competitivas de esta variable. Esa teoría es posible, pero su presentación esta más allá del objetivo de este artículo. Baste decir que establecería una relación entre la tasa de interés y el nivel de los precios y al coste de los servicios bancarios. Del lado de los precios, explicaría la tendencia dominante entre 1947 y 1981, un periodo en el que la tasa de interés nominal creció en paralelo al nivel de precios (como en la "Paradoja de Gibson"). También permitiría intervenciones de política económica concretas, como el llamado "Shock de Volcker" que aumento la tasa de interés del 10,4\% en 1979 al 14,03\% en 1981. Merece la pena recordar que Paul Volcker se convirtió en Presidente de la Reserva Federal solo en agosto de 1979, mientras que las tasas de interés habían estado siguiendo en paralelo al nivel de precios desde hacia tres décadas. Del lado del coste, una teoría como la propuesta explicaría porqué la tasa de interés puede caer de manera relativa al nivel de los precios cuando los costes de los servicios bancarios están cayendo, y puede incluso llegar a desplomarse a pesar de un aumento del nivel de precios, como ocurrió a partir de 1981. Solo entonces podríamos juzgar la influencia relativa de las fuerzas del mercado y de las decisiones políticas en la tendencia de la tasa de interés en la post-guerra.

(16) He subrayado los aspectos esenciales de la cita de Obama. Todas las citas son del informe: "G20 Summit: An Economic Clash of Civilizations", The Christian Science Monitor, 25 de junio de 2010.

(17) "Roosevelt y los halcones inflacionistas del momento estaban decididos ha pinchar lo que consideraban una burbuja bursátil y darle un pellizco a la inflación en el trasero. Equilibrar el presupuesto era un paso importante para ello, pero también la política de la Reserva Federal, que redujo considerablemente la (masa monetaria) al exigir reservas más altas a los bancos. Durante 1937, Roosevelt llevó a cabo su ajuste fiscal a pesar de la obvia recesión de la actividad económica que provocó. El presupuesto fue reequilibrado en el año fiscal de 1938 ...el resultado fue un enorme fracaso económico, con la caída del PIB y el aumento del paro" Bruce Bartlett, "Is Obama Repeating the Mistake of 1937?", Capital gains and Games Blog, 25 de enero de 2010, en www.capitalgainsandgames.com 